\title{
The Evolution of Plug-In Electric Vehicle-Grid Interactions
}

\author{
David P. Tuttle and Ross Baldick
}

\begin{abstract}
Over the past decade key technologies have progressed so that mass-market viable plug-in electric vehicles (PEVs) are now set to reach the first of many major vehicle markets by 2011. PEV-grid interactions comprise a mix of industries that have not interacted closely in the past. A number of these commercial participants have utilized the same basic business model for nearly a century. The various participants include vehicle manufacturers, utilities, and supplier firms who have radically different business models, regulatory and legal environments, geographical scope, and technical capabilities. This paper will provide a survey of PEV technology trends and other factors. From an analysis of these factors this paper synthesizes and provides a likely scenario for PEV-grid interaction over the next decade.
\end{abstract}

Index Terms-Battery electric vehicle (BEV), extended range electric vehicle (eREV), plug-in electric vehicle (PEV), plug-in hybrid vehicle (PHEV), vehicle to grid (V2G).

\section{INTRODUCTION}

$\mathbf{T}$ HERE ARE various motivations for developing alternative energy sources and associated vehicle powertrains to reduce a widespread dependence on oil. The motivations include energy security and its related costs [1], environmental concerns (including climate change and oil spills), air quality [2]-[4], and the costs associated with importing and consuming oil for transportation [5]. A variety of PEV models are emerging, and it is useful to define these, while assessing their strengths and weaknesses. Grid-enabled or plug-in electric vehicles (PEVs) can be broadly categorized into battery electric vehicles (BEVs), extended-range electric vehicles (eREVs), and plug-in hybrid electric vehicles (PHEVs).

BEVs incorporate a large on-board battery, charged while parked via a cord to the power grid. This battery then wholly provides the energy for the electric traction motor to propel the vehicle. eREVs are BEV-derived vehicles with an on-board internal combustion engine (ICE) generator that provides electrical energy to the motor once the initial battery charge is exhausted. This configuration solves the classic "range anxiety" problem of a BEV [6] by providing an overall range on par with a traditional gas or diesel vehicle. Once its initial charge from the grid is depleted, or if the vehicle is never plugged into the grid, the eREV should operate like a conventional hybrid electric vehicles (HEV). PHEVs effectively are HEVs with larger

Manuscript received March 25, 2011; revised July 08, 2011; accepted August 30, 2011. Date of publication January 04, 2012; date of current version February 23, 2012. Paper no. TSG-00111-2011.

The authors are with the Department of Electrical and Computer Engineering, University of Texas at Austin, Austin, TX 78712 USA (e-mail: dave.tuttle@mail.utexas.edu; baldick@mail.utexas.edu).

Color versions of one or more of the figures in this paper are available online at http://ieeexplore.ieee.org.

Digital Object Identifier 10.1109/TSG.2011.2168430 batteries and a charging cord to access grid power. PHEVs typically operate in a "blended" mode, using the gas engine and electric motor together, to substantially reduce gasoline consumption while operating in battery charge depletion (CD) mode [7], [8].

Range-extended (eREV and PHEV) architectures leverage the energy density of petroleum to solve the problem of range anxiety at the cost of incorporating a hybrid electric-gasoline powertrain. Along with the energy density advantage of petroleum, a pervasive refueling infrastructure is leveraged when longer trips are taken. Range-extension capabilities enable the eREVs and PHEVs to serve as a household's primary or sole vehicle. This petroleum-based backup allows downsizing of the most expensive PEV component, the battery (as compared to a $\mathrm{BEV}$ ) while providing a range on par with those of conventional and hybrid-electric vehicles.

Given range anxiety, advances in lithium batteries, and other consumer considerations the mass-market BEVs will likely have 100 mile nominal range targets. An advantage for BEVs is powertrain simplicity and expected very low maintenance costs. As with conventional vehicle mileage estimates, "your actual (electric vehicle) mileage will vary" (typically, but not always in a negative fashion). Real world electric range will vary significantly with driving habits, terrain, and weather conditions given relatively heavy electrical loads such as passenger cabin heating and air conditioning [9].

Electric grid participants consist of generators, transmission and distribution firms, retailers, and, in the near future, aggregators, which are defined here to be are type of retailer that communicates to and controls a sufficiently large number of demand-side resources such as PEVs to effectively create a single controllable resource for the grid operator. All participants are typically motivated by increased vehicle-specific energy sales. Most of these participants also prefer avoiding the aggravation of critical peak demand. If PEVs are charged off-peak, the existing U.S. energy grid can potentially support conversion of $84 \%$ of the light duty vehicles in U.S. to PEVs without significantly added investment [10]. However, if electric vehicle sales increase substantially over time, multiple homes served by a single grid distribution transformer that must solely support the charging of a cluster of new PEVs may force distribution firms to divert a portion of their incremental energy revenues to selectively upgrade these transformers.

To encourage PEV adoption to create this demand, grid participants will be focused on safe, convenient, and cost-effective access to charging stations. In order to avoid aggravating peak demand, grid participants will likely encourage grid friendly charge windows through simple peak/off-peak pricing programs combined with manual driver inputs to the PEV on-board com- 
puter, the offer of subsidized installation of home electric vehicle supply equipment (EVSE, commonly called a charging station) in return for demand response control, or rudimentary signaling of $\mathrm{CO}_{2}$ and prices to the PEV over various communication pathways.

\section{Method And Rationale}

A wide variety of sources from research universities, national labs, standards-setting bodies, and the automotive and utility industries are drawn on to assess the likely progression of technologies, critical priorities and constraints, and business/regulatory environment to identify and articulate a likely scenario of PEV-grid interactions over the course of the next decade. Since the scope of key participants is so broad, subjected to substantially different operating paradigms for many decades, and the technology so new this scenario provides a useful framework for focusing resources to the most potentially relevant areas of research as well placing "a stake in ground" to foster further debate on the likely progression.

\section{RESULTS AND DISCUSSION}

\section{A. First Generation}

First-generation PEVs are available today. The first-generation PEVs will establish "green" technology leadership and build brand equity for their manufacturers, help the vehicle manufacturer meet increasingly strict government fuel economy standards across most regions, drive $\mathrm{R} \& \mathrm{D} /$ manufacturing/supplier base learning, and provide test beds to better understand the durability of batteries and other key components. Volumes are expected to be modest, but meaningful. These PEVs will likely sell at low or negative profit levels depending upon sales volumes, financial accounting for the basic technology R\&D amortization, sales price, tax incentives, and battery warranty costs. The first generation of PEV manufacturing will mainly demonstrate market leadership, while maintaining the extremely high levels of reliability, safety, and convenience that conventional vehicles provide today. Meeting these expectations could be a challenge given PEV technology is new and unproven in large scale customer deployments, which tend to surface problems not easily found despite manufacturers' rigorous validation tests.

An accurate cost-benefit analysis is beyond the scope of this paper and involves considerable effort to determine the purchase price increment between a PEV and a conventional equivalent and then also estimate operating, maintenance, and depreciation costs differences over the life of the vehicle. To provide the most valuable insights to the reader, it is valuable to calculate the net present value using varied gasoline prices and battery replacement costs given the difficulty in projecting these prices over the long term [11]. Depending upon usage patterns, gasoline and electricity prices, and battery replacement, the financial payback can be positive to buyers even in low fuel-cost regions such as the U.S. given substantial tax incentives presently offered [11].

The global vehicle manufacturers likely perceive enough safety and durability risks with these first-generation vehicles that they will avoid including two-way powerflow capability
Fig. 1. Existing SAE J1772 ac level-1/ac level-2 coupler [13].

for the near term. The vast majority of PEVs will likely include only grid-to-vehicle $(\mathrm{G} 2 \mathrm{~V})$ power flow and the driver will have on-board vehicle programmability to manually set the charge window. Modest integrated communication capabilities will be included, which will enable diagnostics and status from the vehicle, such as charge control to set "grid-friendly" charging windows, and control of passenger cabin preheating or precooling.

Unlike the inconvenience and costs associated with the prior generation of two incompatible inductive and conductive chargers, the vehicle manufactures appear to have agreed upon the SAE J1772 conductive charge coupler (Fig. 1) as the standard for upcoming U.S. market vehicles [12]. SAE J1772 presently has two basic charging voltages: single phase ac level-1 $(120 \mathrm{~V})$ and ac level-2 $(240 \mathrm{~V})$ up to a peak power transfer rate of $19.2 \mathrm{~kW}$. This common standard fosters lower charging infrastructure costs, as well as improved availability and convenience of public and home charging stations.

Range extended eREVs and PHEVs typically have smaller (but still considerable) batteries that can charge overnight through a standard ac level-1 $120 \mathrm{~V}$ wall outlet. BEVs will likely need a more expensive ac level-2 $240 \mathrm{~V}$ high-speed charger that may have an installed cost between $\$ 1000$ and $\$ 3000$. Regional efforts to create a large supply of qualified electrical contractors, a streamlined permitting and inspection process, or level-2 charger rebates contingent upon off-peak charging will be useful in substantially driving down the cost of these permanently installed high-speed level-2 chargers in residences. While a meaningful additional up-front cost, these level-2 chargers can be used with vehicles from all manufacturers and will effectively upgrade a home to have its own permanently installed "personal refueling station."

\section{B. Second Generation}

Second-generation PEVs will be developed with far greater amounts of field and lab experience enabling improvements particularly in cost. Enhancements in battery control and efficiency will improve range or maintain range at decreased costs. Hence, second-generation PEVs are likely to have a more attractive financial payback and be somewhat less dependent upon tax incentives. As PEV powertrain components gain scale production economies and become less expensive (or if oil supplies are disrupted or prices increase substantially) relative total cost of ownership improvements will drive further waves of adoption.

Grid to vehicle (G2V) SAE J1772 AC level-1 (120 V) and ac level-2 $(240 \mathrm{~V})$ charging capability will remain but likely be improved with more substantial communication capability such as power line communications (PLC) between the electric vehicle 
supply equipment (EVSE) and PEV, ZigBee ${ }^{\mathrm{TM}}$ wireless communications between the smartmeter and EVSE/PEV, vehicle integrated wireless capability typically over digital cell phone networks, or $802.11 \mathrm{WiFi}^{\mathrm{TM}}$ wireless communications between the PEV and a home area network (HAN). These enhanced communications will enable more sophisticated grid-PEV interactions and more intelligent charging with $\mathrm{CO}_{2}$ /energy-price signaling or perhaps limited regulation-up/regulation-down grid ancillary services, which may generate revenue for the PEV owner. Importantly, additional communications and control capabilities will likely enable more sophisticated charging control which can increase the effective yield and lower costs of incorporating wind, solar, or other intermittent renewable generation sources. The availability of these more sophisticated communications and control are one of the defining characteristics of the second generation. These extra capabilities create the potential for PEVs provide the most flexible, large scale, and effective demand response capability ever developed for the grid. It is likely to take more than 3 to 4 years to have PEV-based grid-advised charging or demand response to be deployed in significant amounts. At the very least, it will take this amount of time to begin to have meaningful numbers of PEVs on the road.

Additional standards efforts (such as SAE International J2847) are underway to enable more sophisticated communications between the PEV, the home area network, and the meters/utility. Also, ac level-2 charging speed may be improved further by increasing the current capability to $30 \mathrm{~A}$ or the maximum $80 \mathrm{~A}$ limit (instead of $240 \mathrm{~V} / 15 \mathrm{~A}$ ) where supported by the premise electrical infrastructure. For example, an increase of maximum level-2 charge rate from $3.3 \mathrm{~kW}$ to 6.6 $\mathrm{kW}$ would reduce the time required for a full $10 \mathrm{kWh}$ eREV charge to about 2 hours.

By the second-generation timeframe, the relative advantages of the various PEV architectures will become increasingly clear to customers, the technology will have advanced further, and costs/performance will likely have been improved. Vehicle manufacturers may have enough knowledge about technologies and PEV consumer behaviors and preferences to offer an increased diversity of vehicle platforms using the same basic electric powertrain components or derivatives. For example, given the strong torque capabilities of electric motors, differentiated performance PEVs will likely be announced. Performance cars traditionally have a higher sales price and provide larger profit margins for vehicle manufacturers. These greater margins can more profitably recover the additional costs of the electric powertrain and batteries by providing the customer with "guilt-free performance." Increased number of PEV types and performance, lower costs, more familiarity with PEVs may then increase PEV adoption rates.

\section{Third Generation}

Third-generation PEVs may be substantially defined as vehicles with two-way powerflow capabilities. This two-way powerflow capability may be combined with the introduction of an industry standard de ultra-fast high-capacity interface to the vehicle (beyond the ac level-2 interface defined today) supporting a higher maximum powerflow of approximately $50 \mathrm{~kW}$ to $100 \mathrm{~kW}$.
The first reverse powerflow configuration may be vehicle to load (V2L) [14]. V2L capability will enable the PEV to act as a construction-site generator to an isolated load. An example of this configuration would be a PEV pickup-truck which would include an on-board charger, converter, and pickup-bed mounted power outlets.

The PEV could act as a home backup generator in a vehicle-to-home (V2H) configuration. Multiple PEVs acting in concert with a local coordinator could support a larger isolated building/mobile command center/Military mobile hospital in a vehicle-to-premise (V2P) configuration. In V2P, there is no coordination with the grid system operator, but instead there is local communication and control of the PEVs as an independent cluster of generation resources not tied to a larger electricity grid.

Given there is little or no coordination needed with the grid, it is possible that some vehicle manufacturers may introduce V2L, V2H, or V2P capability more quickly than fourth-generation PEVs with two-way powerflow and sophisticated coordination with the grid. This timeframe could be after 5 years as major vehicle and battery manufacturers have gained considerable field experience with battery durability, battery costs have declined substantially, after PHEV pickup trucks are introduced in addition to the common small four-door sedan PEV, or projected profitability for this niche vehicle feature becomes financially attractive.

Basic vehicle-to-grid (V2G-net-metered or V2G-NM) interactions could leverage the PEV as a distributed storage node to capture locally generated energy from photovoltaic panels or store low-cost off-peak energy for later release back to the grid at higher peak rates through "net-metering." Net-metering capability enables a home's electric meter to effectively run backward to credit the customer's account when their local generation (such as rooftop solar panels) produce more energy than their home demands. The excess energy is fed back into the grid. Unlike residential photovoltaic panels which may provide excess power back to the grid simply based upon total sunlight available and the local load, the increased communication and control of PEV can provide greater coordination and optimization of reverse power flow to the grid.

Additional configurations of two-way power flow to the grid with both G2V combined with vehicle-to-home/vehicle-to-premise/vehicle-to-grid capability will likely require an off-vehicle EVSE/power outlet/transfer switch designed to meet the required premise building electricity codes (such as "islanding" when the grid power is off) and perhaps an industry standard high-capacity interface if large amounts of energy flow are required.

To recover their incremental $\mathrm{R} \& \mathrm{D}$, manufacturing, and warranty costs the vehicle manufacturers will likely charge an additional premium for a two-way powerflow capable interface and an off-board charger/power outlet/transfer switch box.

\section{Fourth Generation}

With assured two-way communication and control, additional software, and grid aggregators fourth-generation PEVs may be enabled to generate increased revenue (or reduced 
energy costs) for the owner through the use of their onboard battery and gasoline generator.

Fourth-generation PEVs have a greater ability to be used as a source for grid ancillary services or peak power sales back to the grid [15], [16]. PEVs may act as a distributed storage node with their large battery storing less-expensive off-peak energy from the grid or locally generated renewable energy from rooftop solar panels, providing power for the premise, or releasing excess energy back to the grid during higher priced peak demand. Estimates for the revenue potential for the PEV owner from ancillary services vary. A large portion of the variation in estimates appears to be from varied market price assumptions for ancillary services in the different regions, and differences in assumptions on the costs of aggregation and vehicle availability to the aggregators. V2G capability and aggregators would be required to support ancillary services such as regulation up, regulation down, and spinning reserves for the grid independent system operator [17], [18]. The limitations to using the PEV for advanced V2G will likely be related to the challenge of implementing assured and secure communications particularly between the aggregator and the large number of PEVs, the amount of the potential income, the additional wear on the PEV battery, and the degree of inconvenience to the driver. Given these challenges, meaningful adoption of reverse powerflow V2G is likely to take more than 8 to 10 years (approximately two vehicle generations).

The use of PEV range extending engines to generate energy (and create compensating revenues for the PEV owner) which is then fed back to the grid to reduce grid peak demand has questionable likelihood of achieving mass adoption given the complexities of control, and unattractive economics and emissions compared to traditional very large scale grid generation.

Another concept is to use coordinated PEVs as a grid feeder backup. The need for assured communication and the complexity of coordination make the use of PEVs for feeder backup extremely challenging. Orchestration of this concept would require coordinated isolation of the feeder through grid protection and isolation devices such as relays, breakers, and fuses. The concept would likely require a large number of sophisticated coordination activities related to frequency, voltage, reactive power, cold start, feeder configurations, and shutdown.

Significant investment and interest in advanced V2G PEVgrid interactions will likely require policy action or regional specific circumstances which create sufficient financial incentives.

Using PEVs as synergistic grid storage will be more compelling to utilities when new sources of fast ramp/zero- $\mathrm{CO}_{2}$ generation, spinning reserves, or regulation ancillary services are needed to enable greater deployment of intermittent renewable generation. This increased thrust for greater renewable generation and hence sophisticated PEV storage control may be most strongly accelerated by increasing renewable portfolio credits, renewable fuel credits, production tax credits, carbon taxes, or other policy actions.

Total PEV fleet size is likely to not be a meaningful fraction of the total number of vehicles (approximately 240 million) on U.S. highways for a number of years. To assess the likely timeframe when a total of 1 million PEVs would be on U.S. highways, KEMA [17] assumed that the PEV adoption curve for the next ten years would be similar to the Prius adoption rate of the past ten years. Assuming the "target" adoption rate stated in the KEMA study with 1 M PEVs on U.S. highways by 2017 and nearly $2 \mathrm{M}$ by the end of 2020, PEV sales should be approximately $2 \%$ of new vehicle sales by 2020 , nearly equivalent to the $2.5 \%$ hybrid new vehicle market share today in the U.S. Assuming 13 million vehicles sold per year, an estimated 300000 PEVs would be sold yearly in 2020. A further projected breakdown of these $2 \mathrm{M}$ PEVs into BEVs, eREVs, and PHEVs is highly speculative given that the eventual mix may likely be dependent upon many factors. Those factors include the popularity of a particularly styled PEV, brand preference and reputation, one brand and/or type of PEV having better or worse battery durability experiences, technical breakthroughs, oil prices, and battery cost and gasoline range extender cost declines over the course of 9 years. Therefore, any analysis whose outcomes depend upon the specific mix of types of PEVs would benefit from including sensitivity analysis showing the effect of different mixes.

\section{CONCLUSION}

The first generation of mass-market viable PEVs are now available but still in their infancy. PEV-grid capabilities will be defined not only by the rate of technology development but will likely also be guided, accelerated, or limited by the regionally unique financial incentives, regulatory structure and requirements, and values of each participant. It is possible that incremental or breakthrough technology progress may accelerate the progression, but other factors such as communications standards, long vehicle development cycles, and the required gridside communications and control infrastructure may be constraining factors which may keep the progression of PEV-grid interactions in the same approximate order.

Vehicle OEMs are fundamentally driven to create PEVs with compelling design, image, and features that will create profitable vehicle sales. Safety and durability are, of course, also critical and fundamental objectives. The additional software cost to enable "grid friendly" charge window programming is negligible and hence expected to be incorporated into all PEVs. More advanced grid-advised or renewable generation coincident charging can be enabled by relatively simple broadcast of emission or price related information to PEVs. Algorithms programmed into the PEV on-board computer can then deduce the optimal charging profile. With more advanced communications and grid aggregators, the sale of ancillary services such as regulation up/regulation down could produce revenue for the PEV owner by regulating G2V charging of the vehicle.

Enabling basic two-way power flow for V2L, V2H, or V2P adds extra hardware costs, adds risk of stress and failure to PEV components and battery, and increases product liability exposure. An extra cost V2L contractor site generator or V2H/V2P backup generator option that avoids the need for sophisticated external communication and coordination could be profitable for vehicle manufacturers. PEVs enabling V2L, V2H, V2P, or basic V2G-net-metering capability can likely be profitably offered as an extra cost option once sufficient field experience has been gained to understand and address key technology failure mechanisms. Given there are few dependencies upon advanced 
TABLE I

PROGRESSION OF PEV-GRID INTERACTIONS

\begin{tabular}{|c|c|c|c|}
\hline PEV Generation & Power Flow & Communications Characteristics & $\begin{array}{l}\text { PEV-Grid Interaction } \\
\text { Characteristics }\end{array}$ \\
\hline First Generation & Grid-to-Vehicle (G2V) & Over cell phone network (if any) & $\begin{array}{l}\text { G2V with manual driver } \\
\text { programmed "grid friendly" charge } \\
\text { window }\end{array}$ \\
\hline Second Generation & Grid-to-Vehicle (G2V) & $\begin{array}{l}\text { Real-time broadcast of } \mathrm{CO}_{2} \text { and price } \\
\text { information to } \mathrm{PEVs} \\
\text { Grid-to-PEV communications via } \\
\text { aggregator }\end{array}$ & $\begin{array}{l}\text { G2V with advanced intelligent } \\
\text { charging aligned with renewable } \\
\text { generation } \\
\text { G2V with limited regulation up and } \\
\text { regulation down ancillary services }\end{array}$ \\
\hline Third Generation & $\begin{array}{l}\text { Grid-to-Vehicle (G2V) plus Vehicle- } \\
\text { to-Load (V2L) } \\
\text { Grid-to-Vehicle (G2V) plus Vehicle- } \\
\text { to-Home (V2H) } \\
\text { Grid-to-Vehicle (G2V) plus Vehicle- } \\
\text { to-Premise (V2P) }\end{array}$ & $\begin{array}{l}\text { EVSE-PEV communication only (no } \\
\text { external communications) } \\
\text { EVSE-PEV communication only (no } \\
\text { external communications) } \\
\text { EVSE(s)-PEV(s) communication } \\
\text { only (no external communications) }\end{array}$ & $\begin{array}{l}\text { V2L for construction site generator } \\
\text { V2H for home backup generator } \\
\text { (isolated through premise transfer } \\
\text { switch) } \\
\text { V2P as building backup generator } \\
\text { (isolated through transfer switch and } \\
\text { coordinated by a local aggregator) }\end{array}$ \\
\hline & $\begin{array}{l}\text { Grid-to-Vehicle (G2V) plus Vehicle- } \\
\text { to-Grid-Net Metered (V2G-NM) }\end{array}$ & $\begin{array}{l}\text { EVSE-PEV communication only (no } \\
\text { external communications) }\end{array}$ & $\begin{array}{l}\text { V2G-Net-Metered: Local generation } \\
\text { (such as rooftop photovoltaics) with } \\
\text { reverse power flow of excess energy } \\
\text { and net-metering. }\end{array}$ \\
\hline Fourth Generation & $\begin{array}{l}\text { Grid-to-Vehicle (G2V) plus } \\
\text { Advanced Vehicle-to-Grid (V2G- } \\
\text { Advanced) }\end{array}$ & $\begin{array}{l}\text { Assured secure two-way Grid-PEV } \\
\text { communication }\end{array}$ & $\begin{array}{l}\text { V2G-Advanced: Grid Ancillary } \\
\text { Services provided by two-way power } \\
\text { flow of PEV battery energy and/or } \\
\text { local generation (such as rooftop } \\
\text { photovoltaics) }\end{array}$ \\
\hline
\end{tabular}

external communications and control or industry standards development, this option holds promise of commercialization as soon as vehicle manufactures can profitably engineer a sufficiently robust hardware and software solution.

V2G with limited communication could be useful and financially attractive in regions with substantial time-of-use price differentials or premise solar or wind generation which is net-metered back to the grid. Over the next ten years, introduction of the most advanced V2G capability which supports the sale of the most rich set of ancillary services is expected to be limited by the availability of assured PEV-grid communications and two-way power flow capability and control on the PEV. The varied communication and control pathways, reliability requirements, other performance parameters, and financial payback are complex and all areas worthy of further research. Vehicle manufacturers may also be hesitant to offer this capability for a number of years until the wear mechanisms and risks are well known and they understand how to profitably offer this feature.

Grid participants are typically motivated by increased vehicle-specific energy sales while also avoiding the aggravation of critical peak demand. To encourage PEV adoption to create this demand, grid participants will be focused on safe, convenient, and cost effective access to charging stations. Also, in order to avoid aggravating peak demand, grid participants may offer lower cost off-peak tariff programs or subsidizing EVSE installations in return for demand response control. Installation of intelligent EVSEs with demand response may then provide the capability to beneficially align PEV charging with intermittent renewable generation output.

Regionally specific circumstances may create compellingly high prices for ancillary services or other special PEV-grid interactions. Otherwise, the most advanced V2G PEV-grid interactions may require policy actions to foster the needed investments by the relevant participants. An increased policy thrust for deployment of greater amounts of intermittent renewable generation on the grid may provide the needed financial incentives to create the communication and control systems to use PEVs as synergistic grid storage, fast ramping reserves, or for regulation ancillary services.

This paper is an attempt to articulate the most important factors which affect PEV adoption, characteristics, capabilities, and interactions with the grid over the next decade. While there will be differences in the types of PEVs, the evolution of their interactions with the grid is likely to be fairly similar given the required development and investment in grid-side communications and control functions to enable each successive generation of PEVs, and because of the common battery durability knowledge that will be gained from an increasing number of PEVs on the road, and a greater number of years of expertise accumulated. A likely, or at least possible, progression of PEV-grid interactions is synthesized and summarized in Table I. While the timeframes for the introduction of each generation are likely to be different for various regions, a combination of limiting and interdependent factors may prove the progression to be a durable framework across multiple regions. 
In particular, various $\mathrm{G} 2 \mathrm{~V}$ configurations will be developed well before the advent of $\mathrm{V} 2 \mathrm{G}$ and this paper articulates a progression of beneficial PEV-grid interactions.

\section{REFERENCES}

[1] D. L. Greene, "Measuring energy security: Can the United States achieve oil independence?," Energy Policy, vol. 38, no. 4, pp. 1614-1621, 2010.

[2] R. Sioshanshi and P. Denholm, "Emissions impacts and benefits of plug-in hybrid electric vehicles and vehicle to grid services," National Renewable Energy Laboratory, Paper, 2008 [Online]. Available: http://www.iwse.osu.edu/ISEFaculty/sioshansi/papers/PHEV_emissions.pdf

[3] T. Thompson, M. Webber, and D. Allen, "Air quality impacts of using overnight electricity generation to charge plug-in hybrid electric vehicles for daytime use," Environ. Res. Lett., vol. 4, 2009, Art. no. 014002.

[4] "Environmental Assessment of plug-in hybrid electric vehicles; Volume 2: United States air quality analysis based on AEO-2006 assumptions for 2030," Washington, D.C., final rep., Electric Power Research Institute (EPRI) and National Resources Defense Council, 2007 [Online]. Available: http://my.epri.com/portal/server. pt?open $=514 \&$ objID $=223132 \&$ mode $=2$

[5] U.S. BEA, "U.S. international trade in goods and services," U.S. Census Bureau of Economic Analysis, Mar. 11, 2008.

[6] T. Markel, "Plug-in electric vehicle infrastructure: A foundation for electrified transportation ," presented at the MIT Energy Initiative Transp. Electrification Symp., Cambridge, MA, 2010 [Online]. Available: http://www.nrel.gov/docs/fy10osti/47951.pdf, National Renewable Energy Laboratory Conf. Paper 540-47951

[7] A. Vyas, D. Santini, and L. Johnson, "Plug-in hybrid electric vehicles' potential for petroleum use reduction: Issues involved in developing reliable estimates," in Proc. 89th Annu. Meet. Transp. Res. Board, Washington, DC, 2009.

[8] D. P. Tuttle and R. Baldick, "Plug-in electric vehicle-grid interactions," Working Paper, vol. 3, 2010 [Online]. Available: http://users.ece.utexas.edu/ baldick/papers/papers.html

[9] D. P. Tuttle and R. Baldick, "Plug-in electric vehicle-grid interactions," Working Paper [Online]. Available: http://users.ece.utexas.edu/ $\sim$ baldick/papers/papers.html 2010

[10] M. Kintner-Meyer, K. Schneider, and R. Pratt, "Impact assessment of plug-in hybrid vehicles on electric utilities and regional US power grids. Part I: Technical analysis," Pacific Northwest National Laboratory Paper 2007 [Online]. Available: http://www.pnl.gov/energy/eed/ etd/pdfs/phev_feasibility_analysis_combined.pdf

[11] D. P. Tuttle and K. Kockelman, "Electrified vehicle technology trends, infrastructure implications, and cost comparisons," Transp. Res. D, submitted for publication.

[12] SAE International, "SAE standard on EV charging connector approved," retrieved Mar. 14, 2010 [Online]. Available: http://www.sae. org/mags/AEI/7479
[13] SAE International, "SAE's J1772 'combo connector' for ac and dc charging advances with IEEE's help," retrieved Sept 8, 2011 [Online]. Available: http://ev.sae.org/article/10128

[14] R. Scholar, SAE International, in J2847 Committee Meetings, 2009-2010.

[15] W. Kempton and J. Tomic, "Vehicle-to-grid power fundamentals: Calculating capacity and net revenue," J. Power Sources, pp. 1-12, 2005.

[16] A. N. Brooks, "Vehicle-to-grid demonstration project: Grid regulation ancillary service with a battery electric vehicle," pp. 1-12, 2002, Contract number 01-313, prepared for the California Air Resources Board.

[17] ISO/RTO Council and KEMA Inc., "Assessment of plug-in electric vehicle integration with ISO/RTO systems," Dec. 2009.

[18] C. Quinn, D. Zimmerle, and T. Bradley, "The effect of communication architecture on the availability, reliability, and economics of plug-in hybrid electric vehicle-to-grid ancillary services," J. Power Sources, vol. 195, no. 5, pp. 1500-1509, Mar. 2010, V2G ancillary service architecture modeling and analyses, PHEV-2009 conference presentation.

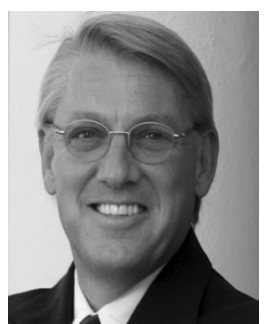

Dave Tuttle (M'06) received the B.S. and M.Eng. degrees in electrical engineering with highest honors from the Speed Scientific School, University of Louisville, Louisville, KY, in 1981 and 1982, respectively, and the M.B.A. degree with the Dean's Award from the University of Texas at Austin in 1991. He is currently working toward the Ph.D. degree at the University of Texas at Austin.

He was responsible for designing microprocessors and leading microprocessor and systems development teams at IBM (1982-2000). He later formed a design team for Sun Microsystems, Austin, focused on multicore/multithread microprocessor development. He is a Research Fellow in the Department of Electrical and Computer Engineering at the University of Texas at Austin. His current research interests are PEVs, PEV interactions and synergies with the electric grid, and renewable energy.

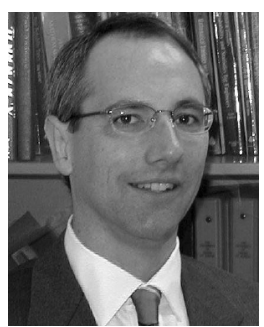

Ross Baldick (F'07) received the B.Sc. degree in mathematics and physics and the B.E. degree in electrical engineering from the University of Sydney, Australia, and the M.S. and Ph.D. degrees in electrical engineering and computer sciences from the University of California, Berkeley, in 1988 and 1990 , respectively.

From 1991 to 1992 he was a Postdoctoral Fellow at the Lawrence Berkeley Laboratory. In 1992 and 1993 he was an Assistant Professor at Worcester Polytechnic Institute. He is currently a Professor in the Department of Electrical and Computer Engineering at the University of Texas at Austin. 